\title{
Potential impact of diabetes prevention on mortality and future burden of dementia and disability: a modelling study
}

\author{
Piotr Bandosz ${ }^{1,2}$ (D) Sara Ahmadi-Abhari ${ }^{3,4}$ - Maria Guzman-Castillo ${ }^{1,5}$ • Jonathan Pearson-Stuttard ${ }^{1,6}$.

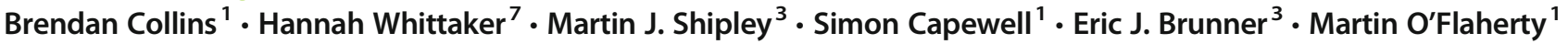

Received: 15 April 2019 / Accepted: 9 August 2019/Published online: 15 November 2019

(C) The Author(s) 2019

\begin{abstract}
Aims/hypothesis Diabetes is associated with an increased risk of dementia. We estimated the potential impact of trends in diabetes prevalence upon mortality and the future burden of dementia and disability in England and Wales.

Methods We used a probabilistic multi-state, open cohort Markov model to integrate observed trends in diabetes, cardiovascular disease and dementia to forecast the occurrence of disability and dementia up to the year 2060. Model input data were taken from the English Longitudinal Study of Ageing, Office for National Statistics vital data and published effect estimates for health-state transition probabilities. The baseline scenario corresponded to recent trends in obesity: a 26\% increase in the number of people with diabetes by 2060 . This scenario was evaluated against three alternative projected trends in diabetes: increases of $49 \%$, $20 \%$ and $7 \%$.

Results Our results suggest that changes in the trend in diabetes prevalence will lead to changes in mortality and incidence of dementia and disability, which will become visible after 10-15 years. If the relative prevalence of diabetes increases $49 \%$ by 2060, expected additional deaths would be approximately 255,000 (95\% uncertainty interval [UI] 236,000-272,200), with $85,900(71,500-101,600)$ cumulative additional cases of dementia and 104,900 (85,900-125,400) additional cases of disability. With a smaller relative increase in diabetes prevalence (7\% increase by 2060), we estimated 222,200 (205,700-237,300) fewer deaths, and 77,000 (64,300-90,800) and 93,300 (76,700-111,400) fewer additional cases of dementia and disability, respectively, than the baseline case of a $26 \%$ increase in diabetes.

Conclusions/interpretation Reducing the burden of diabetes could result in substantial reductions in the incidence of dementia and disability over the medium to long term.
\end{abstract}

Keywords Dementia $\cdot$ Diabetes $\cdot$ Disability $\cdot$ Forecast $\cdot$ Modelling study

Eric J. Brunner and Martin O'Flaherty are joint senior authors

Electronic supplementary material The online version of this article (https://doi.org/10.1007/s00125-019-05015-4) contains peer-reviewed but unedited supplementary supplementary material, which is available to authorised users.

Piotr Bandosz

P.Bandosz@liverpool.ac.uk

1 Department of Public Health and Policy, University of Liverpool, 3rd Floor, Whelan Building, Brownlow Hill, Liverpool L69 3GB, UK

2 Department of Prevention and Medical Education, Medical University of Gdansk, Gdansk, Poland

3 Institute of Epidemiology and Health Care, University College London, London, UK
4 Ageing Epidemiology (AGE) Research Unit, Imperial College London, London, UK

5 Department of Social Sciences, University of Helsinki, Helsinki, Finland

6 School of Public Health, Imperial College London, London, UK

National Heart and Lung Institute (NHLI), Imperial College London, London, UK 


\section{Research in context}

\section{What is already known about this subject?}

- Diabetes increases the risk of developing dementia and disability

- The prevalence of type 2 diabetes has increased globally, alongside the increasing epidemic of obesity

- There are complex inter-relationships between the projected ageing population in England and decreasing incidence of cardiovascular disease and dementia, which are not accounted for in existing studies

\section{What is the key question?}

- What is the potential impact of trends in diabetes prevalence upon mortality and future burden of dementia and disability in England and Wales?

What are the new findings?

- Reducing the burden of diabetes could result in considerable reductions in incidence of dementia and disability

- This impact could be substantial, but would not be felt fully for some decades

How might this impact on clinical practice in the foreseeable future?

- Public health interventions aimed at decreasing the incidence of diabetes may contribute to decreasing the future incidence of dementia and disability, but only after a time lag measured in decades

$\begin{array}{ll}\begin{array}{l}\text { Abbreviations } \\ \text { CVD }\end{array} & \text { Cardiovascular disease } \\ \text { DPM } & \text { Diabetes Prevalence Model } \\ \text { ELSA } & \text { English Longitudinal Study of Ageing } \\ \text { IMPACT-BAM } & \text { IMPACT Better Ageing Model } \\ \text { LYG } & \text { Life years gained } \\ \text { PAR } & \text { Population attributable risk } \\ \text { PARF } & \text { Population attributable risk fraction } \\ \text { PHE } & \text { Public Health England } \\ \text { UI } & \text { Uncertainty interval }\end{array}$

\section{Introduction}

The rapid ageing of populations in high-income countries has triggered major concerns regarding the future burden of agerelated chronic diseases. In England and Wales, there are around 800,000 people living with dementia, and this number is set to increase by $60 \%$ by 2040 [1]. A substantial increase is also predicted for the number of people living with any functional impairment (disability). This constitutes a major policy challenge [2].

Currently, there is no dementia treatment that can significantly modify disease progression. However, several modifiable risk factors for dementia and disability have been identified, thus creating the possibility for prevention. Up to $35 \%$ of cases of dementia are attributable to a combination of nine risk factors: low educational attainment, midlife hypertension, midlife obesity, hearing loss, late-life depression, diabetes, physical inactivity, smoking and social isolation [3].
Reducing these risk factors can have a substantial impact on the risk of developing dementia. During 25 years of follow-up in a large cohort study in the USA, dementia incidence was approximately $80 \%$ higher in those with diabetes compared with those without diabetes [4]. Among individuals in late midlife, those with diabetes had a $24 \%$ faster cognitive decline than those without diabetes [5]. For disability, the Global Burden of Disease Study showed that diabetes was the sixth most common cause of disability in 2015 [2]. England and Wales have recently experienced favourable declines in smoking prevalence and BP levels. These possibly explain the $2.7 \%$ annual decrease in dementia incidence observed in the last two decades [1]. However, there is evidence of worsening trends in other, potentially important, risk factors for both dementia and disability. The prevalence of adult obesity increased from $15 \%$ in 1993 to $26 \%$ in 2010 before recently plateauing [6]. Type 2 diabetes prevalence, driven mainly by obesity trends, more than doubled between 1993 and 2015 and is expected to increase further from $8.6 \%$ in 2015 to $9.7 \%$ by 2035 , generating more than 1.1 million additional cases in England [7, 8]. Midlife obesity and type 2 diabetes have both been shown to be related to dementia and disability incidence, based on evidence from observational studies $[2,9,10]$.

The UK government has responded with prevention efforts to reduce the burden of obesity and diabetes. These include the Diabetes Prevention Programme which targets individuals at highest risk. There have also been population-based policy approaches to prevent obesity such as the soft drinks industry levy, sugar and 'calorie' reduction programmes, and national marketing campaigns [11, 12]. Despite these efforts, future trends in obesity and diabetes prevalence remain uncertain. 


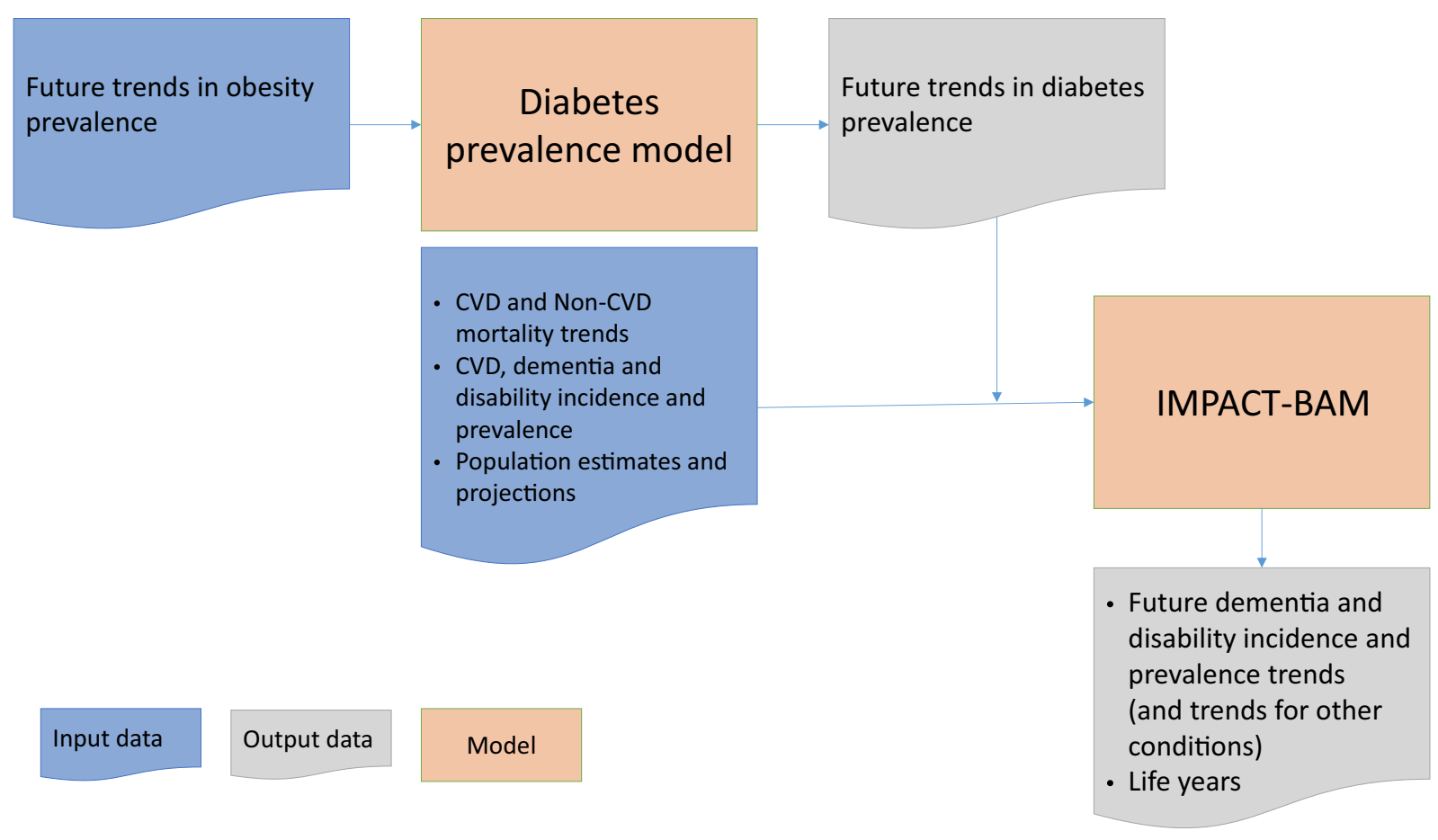

Fig. 1 Diagram illustrating steps of the analysis

Correspondingly, the burdens of dementia and disability, including mortality rates, are unknown.

In this study, we aimed to estimate the potential effects of future trends in diabetes prevalence on mortality outcomes and the future burden of dementia and disability in England and Wales up to 2060.

\section{Methods}

We conducted our analyses in two stages: first, we estimated plausible future trends in diabetes prevalence given different futures for the obesity trends using the Diabetes Prevalence Model (DPM) developed by Public Health England (PHE).

Second, we estimated future trends in diabetes prevalence and examined their potential effects upon dementia and disability over the period 2015-2060 in the England and Wales population using our previously validated IMPACT Better Ageing Model (IMPACT-BAM) [1, 13]. The flowchart in Fig. 1 describes how these two steps are connected.

\section{Future diabetes trends}

In the first step, we examined four plausible future diabetes prevalence scenarios based on obesity prevalence projections. We assumed different futures for the currently increasing obesity trends - no change, a further acceleration, a halt, and finally, a reverse - and translated these into expected future trends in diabetes prevalence using the DPM [7]. We used this model to estimate possible future trends in diabetes prevalence given the four different futures for the currently increasing obesity trends, ranging from a 5 yearly change in the obesity prevalence of -3 to $+5 \%$. Each one of the projected trends in diabetes prevalence from 2015-2060 was used as a different scenario to be explored later in IMPACT-BAM (see electronic supplementary material [ESM] Chapter 4.4 and ESM Fig. 12).

The baseline scenario assumed a continuation of recent trends in leading diabetes drivers, mainly an increase in obesity prevalence of $1 \%$ every 5 years. This obesity trend corresponds roughly to the last 5 years of change in obesity reported in the Health Survey for England study [8]. For this trend, the DPM predicted a $26 \%$ rise in diabetes prevalence from $8.6 \%$ in 2015 to $10.8 \%$ in 2060 .

Three additional scenarios, based on current trends in drivers of diabetes (a further acceleration, a halt, and a reverse in the current obesity trend) were evaluated against the baseline scenario. These scenarios were: $(\mathrm{A})$ an increase in relative diabetes prevalence of $49 \%$; (B) an increase by $20 \%$; and (C) a slowing down of the increase to $7 \%$ by 2060 . Details of all modelled scenarios are presented in Table 1 and ESM Chapter 4.4.

\section{Effect of diabetes trends in dementia and disability}

Overview of the IMPACT-BAM model We extended the IMPACT-BAM to estimate the potential effects of the plausible changing trends in diabetes prevalence upon dementia and disability over the period 2015-2060 in the England and Wales population. 
Table 1 Predicted prevalence of type 2 diabetes by calendar year for four scenarios of trends in obesity, compared with the baseline scenario

\begin{tabular}{|c|c|c|c|c|c|c|c|c|}
\hline \multirow[t]{3}{*}{ Scenario } & \multirow{3}{*}{$\begin{array}{l}\text { Change in obesity } \\
\text { prevalence } \\
(\% / 5 \text { years })\end{array}$} & \multirow{3}{*}{$\begin{array}{l}\text { Change in diabetes } \\
\text { prevalence by } 2060 \\
(\%)\end{array}$} & \multicolumn{6}{|c|}{ Calendar year } \\
\hline & & & 2015 & 2020 & 2030 & 2040 & 2050 & 2060 \\
\hline & & & \multicolumn{6}{|c|}{ Diabetes prevalence $(\%)$} \\
\hline A & +5 & +49 & 8.6 & 9.1 & 10.1 & 11.1 & 12.0 & 12.8 \\
\hline Baseline $^{\mathrm{a}}$ & +1 & +26 & 8.6 & 9.0 & 9.6 & 10.1 & 10.5 & 10.8 \\
\hline B & 0 & +20 & 8.6 & 8.9 & 9.5 & 9.9 & 10.2 & 10.3 \\
\hline $\mathrm{C}$ & -3 & +7 & 8.6 & 8.8 & 9.1 & 9.3 & 9.3 & 9.2 \\
\hline
\end{tabular}

Projection of diabetes prevalence based on estimates from PHE DPM using different assumptions of future obesity trends [7]

${ }^{\text {a }}$ Baseline scenario is that the current trend in the prevalence of obesity will continue
IMPACT-BAM is a probabilistic multi-state, open cohort Markov model which follows the progression of a healthy population (aged $\geq 35$ years) of England and Wales from 2006 to 2060 into eight different health states characterised by the presence or absence of cardiovascular disease (CVD), cognitive impairment, dementia and moderate to severe disability, and two states for death from CVD and non-CVD causes (ESM Fig. 1).

Before running the simulation, we populated each state in the model based on the Office for National Statistics population estimates in 2006 (start year) and prevalence of the above conditions from the English Longitudinal Study of Ageing (ELSA), except for the new cohort of 35-year-olds that enters the system through the disease-free state. The simulation allows individuals to move to other states in the model. The arrows in ESM Fig. 1 indicate the possible movements of people between these ten states, which are governed by one-year probabilities of transition. For example, a healthy 55-year-old man starts the simulation in state 1 (disease-free state) in 2006. He moves to state 2 (CVD) in 2007 after having a stroke. In 2008 he could either die from complications of the stroke (he moves to state 9), die from any other causes (he moves to state 10), or he could develop cognitive impairment (moving to state 3 ) or disability (moving to state 5). As above, movements to any state are driven by transition probabilities.

Transition probabilities were previously calculated using combined data from the ELSA and mortality projections. These projections were estimated separately for cardiovascular and non-cardiovascular mortality rates based on observed data reported by the Office for National Statistics up to 2016. P-spline smoothed lines [14-16] were fitted to logarithmic transformed CVD and non-CVD mortality rates in each 5year age band from 1990 to 2016 by sex using the p-spline function in Stata software. Additional details are provided in the ESM Chapter 2 and ESM Figs 2-5.

We defined disability as the inability to independently carry out one or more activities of daily living, which included getting in or out of bed, walking across a room, bathing or showering, using the toilet, dressing, cutting food and eating. This definition of disability captures individuals who have difficulty maintaining independence and require supportive care.
Dementia was defined on the basis of the coexistence of cognitive impairment and disability, or a report of a doctor diagnosis of dementia by the participant or caregiver. Cognitive impairment was defined as an impairment in two or more functional tests (such as orientation to time, immediate and delayed memory, verbal fluency and numeracy function) or a score higher than 3.6 on the Informant Questionnaire on Cognitive Decline (IQCODE) [17].

A more detailed overview of the model can be found in the previously published papers $[1,13]$ and ESM Chapters $1-2$ and ESM Figs 1-5. Information about model validation is provided in ESM Chapter 3 and Figs 6-9. A general description of these types of Markov models can be found in the paper by Briggs and Sculpher [18].

Diabetes trends effect on transition probabilities For this study, we assumed that trends in the prevalence of type 2 diabetes from each scenario, calculated in the first stage, would modify some of the IMPACT-BAM transition probabilities, which ultimately would result in changes in the burden of dementia and disability. We assumed that the affected transition probabilities were those representing the risk of CVD and non-CVD death, cognitive decline incidence, CVD incidence, disability incidence and recovery from disability. To model these changes in transition probabilities, we used an approach based on the population attributable risk fraction (PARF). The PARF calculates the proportion by which disease burden would be reduced if there were no diabetes in the population. A simpler version of this approach has been used previously in other IMPACT models [19, 20].

Previous research has demonstrated that the association between diabetes and cognitive decline and CHD is dependent upon diabetes duration [21]. This is consistent with the view that the presence of vascular risk factors and insulin resistance in midlife increase the risk not only of developing diabetes and atherosclerosis but also of cognitive decline and dementia later in life. The development of hyperglycaemia, glucose intolerance, microvascular and macrovascular complications that are associated with type 2 diabetes then further accelerate diabetes-associated cognitive decline. 
To account for the effect of diabetes duration, we used the extended formula of PARF to account for multi-category exposures [22]. The different categories of exposure represent six different categories of lengths of time living with the disease: diabetes duration of fewer than 5 years; 5-9 years; 10-14 years; 15-19 years; 20-24 years and more than 25 years.

The extended PARF formula requires: (1) age- and sexspecific estimates of the risk factor prevalence at each exposure level; and (2) RRs comparing every exposure level with the unexposed group (i.e. stratified by age, sex and affected transition probability). We used the 2014 Health Survey for England (HSE) data to obtain age- and sex-specific distributions of diabetes prevalence across the six categories of diabetes duration. For the baseline scenario, we assumed that the prevalence in each of the categories would remain constant in the future. For the rest of the scenarios, we modified the prevalence in each category across time to match the ageing of the population (more details are provided in ESM Chapter 4.5 and Figs 13-20) We obtained the RRs from published studies and by carrying out meta-analyses (see ESM Chapter 4, ESM Figs 10-11 and ESM Table 1). All RRs used had been adjusted for BMI. Then, we adjusted these RRs by diabetes duration using the results from the ADVANCE trial, which quantifies the risk for macro- and microvascular complications and allcause death for each 5 year increase in diabetes duration [23].

Ultimately, we were interested in how the PARF changes because of trends in diabetes prevalence. Symbolically, this would be:

$\triangle \mathrm{PARF}=\mathrm{PARF}-\mathrm{PARF}^{\prime}$

where $\mathrm{PARF}^{\prime}$ is the calculated PARF for the (lower) diabetes prevalence from the baseline scenario.

Outcomes For each scenario, the $\triangle \mathrm{PARF}$ was then multiplied by the appropriate transition probability to generate a new transition probability. We repeated this procedure for all the transition probabilities. We then recalculated the IMPACT model with the new set of transition probabilities and generated scenario-specific numbers of cases of dementia, disability and deaths from 2016 onwards and compared them against the baseline scenario (i.e. the model with the original transition probability values).

Finally, we reported the cumulative number of new cases of dementia, disability and deaths attributable to each scenario for the population aged 65 and over, in comparison with the baseline scenario. We also presented life years gained (LYG) for each scenario and the proportion of life spent with disability.

Sensitivity analysis To explore the impact of parameter uncertainty on model outputs, we conducted a probabilistic sensitivity analysis using Monte Carlo simulation. The procedure entailed iterative sampling from specified distributions for the input parameters that were used in the model, and then recalculation of the outputs. We performed 1000 iterations to estimate $95 \%$ uncertainty intervals (95\% UIs) for the output variables.

The model was developed as a package in $\mathrm{R}$ software (version 3.4.2, https://cran.r-project.org). Detailed information on preparing scenarios data and methods used to evaluated the effect of diabetes on model transition probabilities can be found in ESM Chapters 4-6 and ESM Figs 10-20.

\section{Results}

\section{Baseline scenario}

Our IMPACT-BAM baseline results (see Table 2) suggested that the number of disability incident cases among the population aged 65 and over is expected to increase from approximately 223,600 (95\% UI 219,200-228,100) per year in 2015 to $288,100(275,900-300,200)$ in 2045 and then fall slightly to $271,600(253,900-287,400)$ in 2060. The number of incident dementia cases is also expected to rise from approximately $142,000(137,000-147,300)$ per year in 2015 to 208,500 $(197,400-218,900)$ in 2045 and 203,700 $(189,100-217,500)$ in 2060 .

This increase in the number of annual incident cases occurred despite a continuous decline in the incidence rates of dementia and disability. This suggests that the reason for the increasing number of projected new cases of dementia and disability is the projected ageing of the population, due to increasing longevity. The incidence of disability expressed per 1000 population aged $\geq 65$ is forecasted to decrease from approximately 21.4 (95\% UI 21.0-21.9) per 1000 personyears in 2015 to $17.1(16.6-17.6)$ in 2045 and then 13.9 (13.3-14.4) in 2060. This decrease is also observed for dementia, with an expected fall from 13.6 (13.1-14.1) per 1000 person-years in 2015 to $12.4(11.8-12.9)$ in 2045 and 10.4 (9.9-10.9) in 2060.

The projected total mortality rate in people aged $\geq 65$ is predicted to decrease by approximately $39 \%$ between 2015 and 2060, from 38.7 (95\% UI 38.3-39.2) to 23.6 (22.725.0) per 1000 population.

\section{Comparison of three future scenarios}

Effect of diabetes trends on projected mortality For the most adverse scenario (A) we might expect about 79,700 (95\% UI $72,500-86,000)$ additional deaths attributable to diabetes by 2045 (43.4 deaths per 100,000 population) and approximately 255,000 (236,000-272,200) additional deaths (79.5 per 100,000 ) by 2060 (Table 3 ). This corresponds to 
Table 2 Projected number and rate of incident cases of disability, dementia and mortality in the England and Wales population aged $\geq 65$ in 2030,2045 and 2060, compared with those observed in 2015 for the baseline scenario

\begin{tabular}{|c|c|c|c|c|c|c|c|}
\hline \multirow[t]{2}{*}{ Sex } & \multirow[t]{2}{*}{ Year } & \multicolumn{2}{|c|}{ Disability incident cases } & \multicolumn{2}{|c|}{ Dementia incident cases } & \multicolumn{2}{|l|}{ Total deaths } \\
\hline & & Number (thousands) & Per 1000 population & Number (thousands) & Per 1000 population & Number (thousands) & Per 1000 population \\
\hline \multirow[t]{4}{*}{ All } & 2015 & 224 (219 to 228$)$ & 21.4 (21.0 to 21.9$)$ & 142 (137 to 147$)$ & $13.6(13.1$ to 14.1$)$ & 404 (401 to 408$)$ & 38.7 (38.3 to 39.2$)$ \\
\hline & 2030 & 269 (261 to 276$)$ & 19.3 (18.8 to 19.8$)$ & 187 (179 to 194$)$ & 13.4 (12.9 to 13.9$)$ & 442 (436 to 449$)$ & 31.7 (30.9 to 32.6$)$ \\
\hline & 2045 & 288 (276 to 300$)$ & 17.1 (16.6 to 17.6$)$ & 209 (197 to 219$)$ & $12.4(11.8$ to 12.9$)$ & 471 (466 to 478$)$ & $27.9(27.0$ to 29.1$)$ \\
\hline & 2060 & 272 (254 to 287$)$ & $13.9(13.3$ to 14.4$)$ & 204 (189 to 218 ) & $10.4(9.9$ to 10.9$)$ & 462 (458 to 468$)$ & $23.6(22.7$ to 25.0$)$ \\
\hline \multirow[t]{4}{*}{ Men } & 2015 & $100(96.1$ to 104$)$ & $21.2(20.3$ to 22.1$)$ & 61 (57 to 66$)$ & $13.0(12.0$ to 14.0$)$ & 192 (190 to 194$)$ & $40.6(40.0$ to 41.1$)$ \\
\hline & 2030 & $126(120$ to 132$)$ & $19.4(18.4$ to 20.3$)$ & $86(80$ to 92$)$ & $13.2(12.2$ to 14.3$)$ & $217(214$ to 220$)$ & 33.3 (32.5 to 34.3$)$ \\
\hline & 2045 & 137 (128 to 146$)$ & 17.1 (16.1 to 18.1$)$ & 99 (91 to 107$)$ & $12.3(11.3$ to 13.3$)$ & 233 (230 to 236$)$ & $29.0(27.9$ to 30.2$)$ \\
\hline & 2060 & 132 (120 to 142$)$ & $13.8(12.8$ to 14.8$)$ & $99(88$ to 109$)$ & $10.4(9.4$ to 11.4$)$ & $232(230$ to 235$)$ & 24.3 (23.4 to 25.7$)$ \\
\hline \multirow[t]{4}{*}{ Women } & 2015 & 123 (121 to 126$)$ & $21.6(21.2$ to 22.0$)$ & $81(79$ to 83$)$ & 14.1 (13.8 to 14.5$)$ & 213 (211 to 215$)$ & $37.2(36.8$ to 37.7$)$ \\
\hline & 2030 & 143 (139 to 147$)$ & $19.2(18.8$ to 19.7$)$ & $101(97$ to 104$)$ & $13.6(13.1$ to 14.0$)$ & 225 (222 to 229$)$ & $30.3(29.5$ to 31.2$)$ \\
\hline & 2045 & 151 (145 to 156$)$ & 17.1 (16.6 to 17.5$)$ & $110(105$ to 114$)$ & $12.4(11.9$ to 12.9$)$ & 238 (236 to 242 ) & $26.9(26.1$ to 28.1$)$ \\
\hline & 2060 & $140(132$ to 147$)$ & $14.0(13.6$ to 14.3$)$ & 105 (99 to 110$)$ & $10.5(10.1$ to 10.9$)$ & 230 (228 to 233$)$ & $22.9(22.1$ to 24.3$)$ \\
\hline
\end{tabular}

The baseline scenario is that the current trend in the prevalence of diabetes will continue, which will result in a $26 \%$ increase in diabetes prevalence by 2060

95\% UIs are shown in brackets

approximately $650,000(580,000-700,000)$ cumulative life years lost by 2045 and $2,570,000(2,350,000-2,770,000)$ by 2060 , which is $0.9 \%$ of life years lost for 2060 in comparison with the baseline scenario.

In contrast, for scenario B we might expect about 18,900 (95\% UI 17,200-20,400) fewer deaths due to diabetes by 2045 and $58,600(54,300-62,600)$ by 2060 in comparison with the baseline scenario. This resulted in projected LYG of $150,000(140,000-170,000)$ and $600,000(550,000-650,000)$ by 2045 and 2060 , respectively. This corresponds to a $0.2 \%$ increase in the number of life years in comparison with the baseline scenario in 2060 .

For the most optimistic scenario (C), we might expect approximately 73,000 (95\% UI 66,500-78,800) and 222,200 $(205,700-237,300)$ fewer deaths in comparison with the baseline scenario, for 2045 and 2060, respectively (Table 3 ). This results in gaining approximately $600,000(540,000-660,000)$ additional life years by 2045 and 2,290,000 $(2,090,000$ $2,470,000$ ) life years by 2060 (Fig. 2), an increase in life years of $0.8 \%$ in comparison with the baseline scenario in 2060 .

Effect of diabetes trends on disability and dementia Our results suggest that any changes in diabetes prevalence will have almost no effect on dementia and disability burden in the next 5 years (see Table 4). However, these effects increase significantly during subsequent decades. For scenario A (49\% increase in diabetes up to 2060), we might expect approximately 104,900 (95\% UI 85,900-125,400) additional cases of disability and some $85,900(71,500-101,600)$ additional cases of dementia before 2060. On the other hand, if there is a deceleration in the rate of increase of diabetes prevalence (scenario B), we might avoid approximately 24,400 $(20,000-29,200)$ new cases of disability and 20,100 $(16,800-23,700)$ new cases of dementia cumulatively by 2060.

The most optimistic scenario (C) might result in approximately 93,300 $(76,700-111,400)$ fewer cumulative new cases of disability and 77,000 (64,300-90,800) fewer cases of dementia by 2060 . This corresponds to $2.2 \%$ fewer new cases of dementia and $1.9 \%$ fewer cases of disability in comparison with the baseline scenario in 2060 .

Compression of morbidity The projected trends in diabetes prevalence appeared to affect the proportion of life years spent with disability. The percentage of life years in the population spent with disability was $0.4 \%$ lower for scenario C (most optimistic) than the baseline scenario, and $0.9 \%$ lower than scenario A (most pessimistic) (Fig. 3).

\section{Discussion}

\section{Key findings}

Our study suggests that a reduction in diabetes prevalence would result in a decline in age-specific incidence of dementia and disability and postpone these conditions to later years in life. However, trends in the incidence of dementia and disability at the population level would not be observed for a decade as a result of decreases in mortality and prolongation of life. 


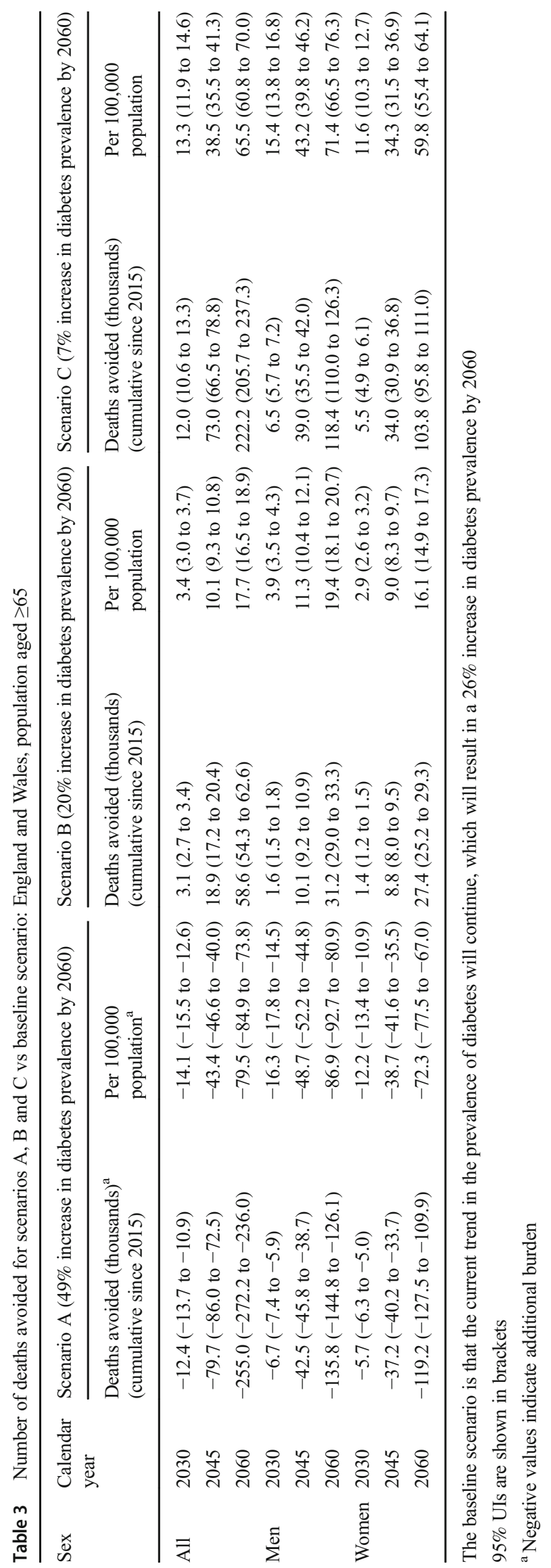




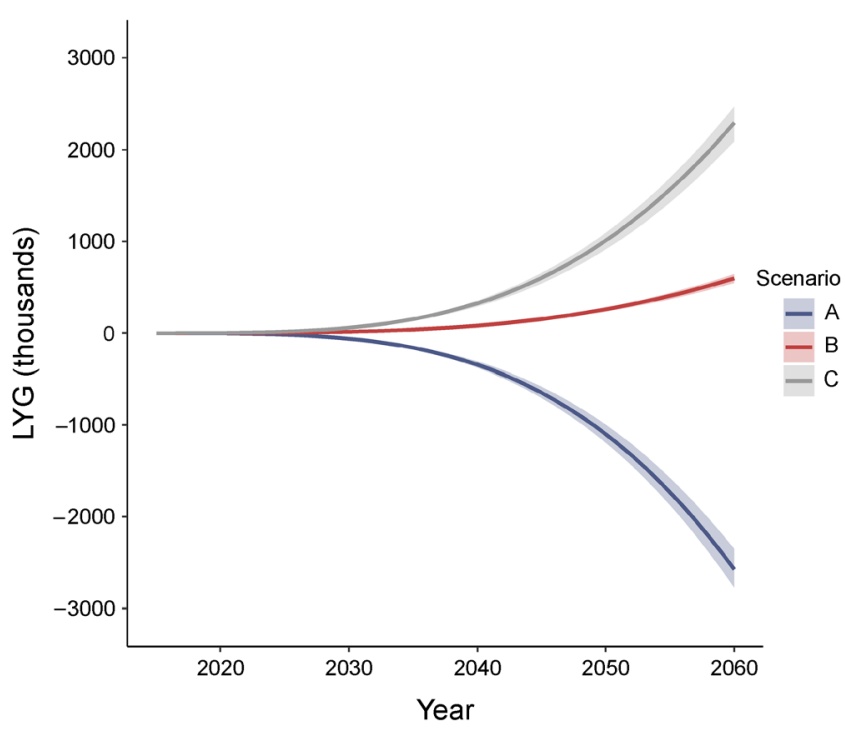

Fig. 2 Cumulative number of LYG for modelled scenarios (thousands) for England and Wales, population aged $\geq 65$. The shading represents $95 \%$ UIs. Scenarios: A, $49 \%$ increase in diabetes by 2060 ; B, $20 \%$ increase in diabetes by $2060 ; \mathrm{C}, 7 \%$ increase in diabetes by 2060

Crucially, our study also suggests that substantial reductions in diabetes prevalence would compress morbidity, decreasing the percentage of life spent with disability by approximately $0.4 \%$ (see Fig. 3).

Our study is the first, to our knowledge, to estimate the potential effect of future diabetes trends on the future UK burden of disability and dementia while taking into account the complex population dynamics of morbidity and mortality.

Barnes and Yaffe [24] estimated the fraction of cases of Alzheimer's disease attributable to diabetes by calculating its population attributable risk (PAR) as $2 \%$ worldwide and $3 \%$ in the USA. This corresponds to 81,000 and 17,000 fewer cases of dementia each year worldwide and in the USA, respectively, if diabetes prevalence decreases by $10 \%$. Our results are far more conservative. It is likely that Barnes and Yaffe's analysis over-estimated the potential burdens, because it did not account for any observed trends in risk factors, mortality or dementia, nor consider competing risks of mortality from CVD as opposed to dementia. Moreover, these analyses, based on simple PAR approaches, did not account for any relationship between diabetes duration and outcomes. Several studies have reported that the RR of fatal CHD increases in individuals with diabetes as the years lived with the disease increases $[21,23,25]$.

\section{Strengths and limitations}

The IMPACT-BAM model accounts for complex epidemiological interactions between several important sources of morbidity like CVD, dementia and disability, which share some risk factors. The structure of the model allowed us to take into account the risks between these conditions and competing risks of dying from cardiovascular and noncardiovascular causes. Diabetes increases the risk of dying, not only from CVD, but also from many other diseases, including several cancers. Given the declining population risk of CVD mortality in future years, the competing risk of nonCVDs is expected to become critical. Our model accounts for this by separating projections of future cardiovascular and non-cardiovascular risk of death, which represents a substantial improvement on previous methodologies [24, 26]. Specifically, we account for trends in CVD- and non-CVD mortality and incidence, trends in dementia incidence, competing risks between cardiovascular and noncardiovascular risk factors. Our methods also account for the increase in risk of both CVD and dementia caused by the time spent living with diabetes. IMPACT-BAM also models the time lag between newly developed diabetes and its complications. To our knowledge, there are no previous studies modelling the effect of prevention of diabetes on dementia and disability which also take into consideration the time needed to develop diabetes complications. This makes our estimates closer to real-life settings. Finally, our prediction model estimates the positive and negative effects of changes in diabetes prevalence. Our scenarios of possible future diabetes burden were based on reasonable assumptions of future obesity trends.

Our study also has limitations. Our approach does not account for any effect of change in the obesity distribution in the population not mediated through diabetes, including the effect on hypertension prevalence. This means that our results may be conservative. However, obesity is a less strong risk factor for dementia than diabetes [4].

We used the pre-existing model published by PHE, the DPM, to calculate expected trends in diabetes prevalence if the obesity prevalence increase accelerates (scenario A), stops (scenario B) or reverses (scenario C). Although obesity is widely recognised as the main driver of the current diabetes epidemic, diabetes trends can also be driven by many other risk factors that may have contrasting trends such as physical activity or pharmacological intervention in individuals with impaired glucose tolerance [27]. In addition, the DPM model (available on PHE official website) lacks information on any potential methodological limitations. Moreover, our approach to projecting the future distribution of the diabetes duration in the population does not account for increased risk of death due to longer duration of diabetes. This can lead to overestimation of the number of participants with longer duration of diabetes, and overestimating the total effect of a change in diabetes prevalence on dementia and disability incidence.

We used a multi-exposure, PARF approach to translate trends in diabetes prevalence to changes in the risk of dementia and cognitive impairment. This approach assumes causality of risk factor and disease. The evidence is still not as strong as it is for the association between some other risk factors and 


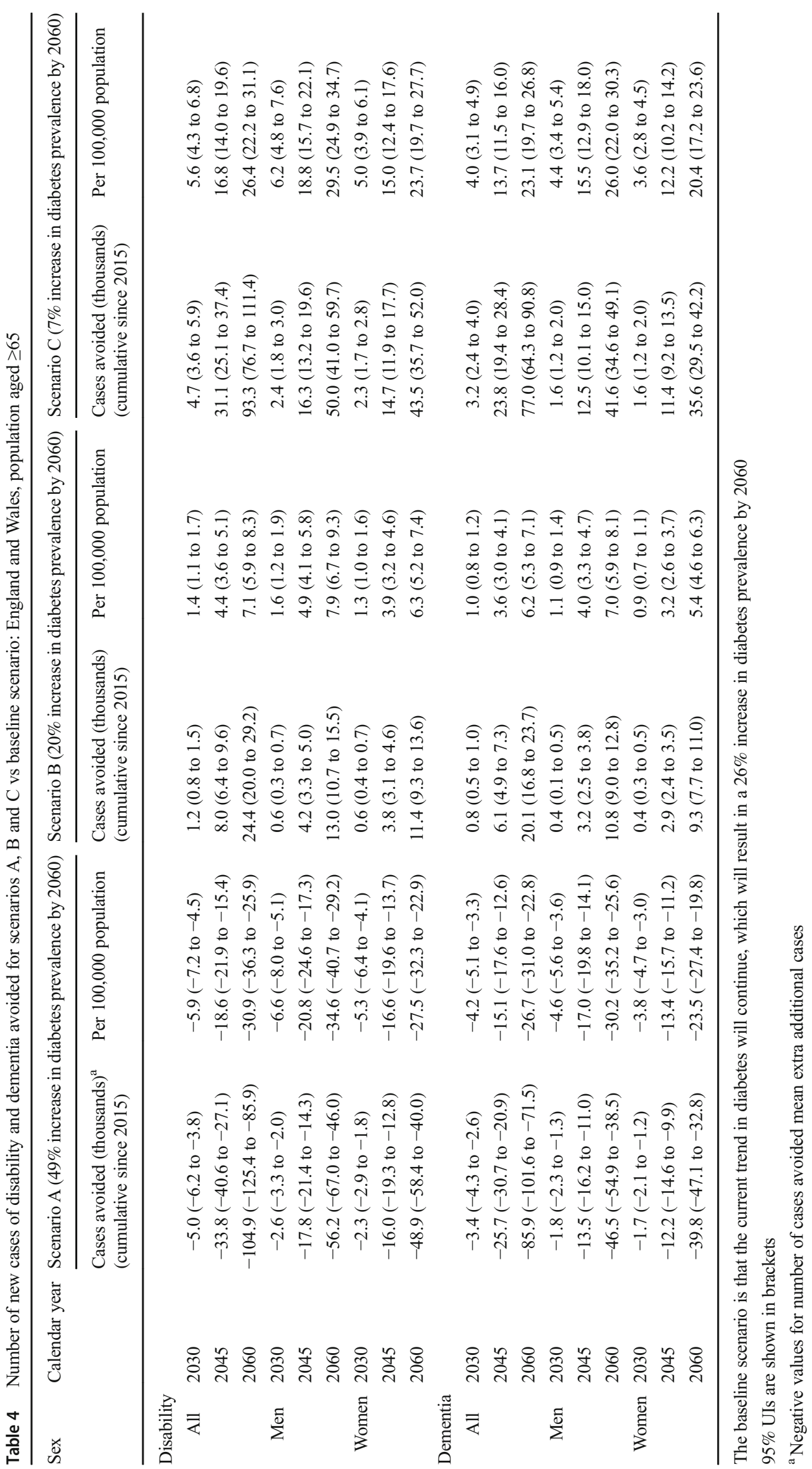




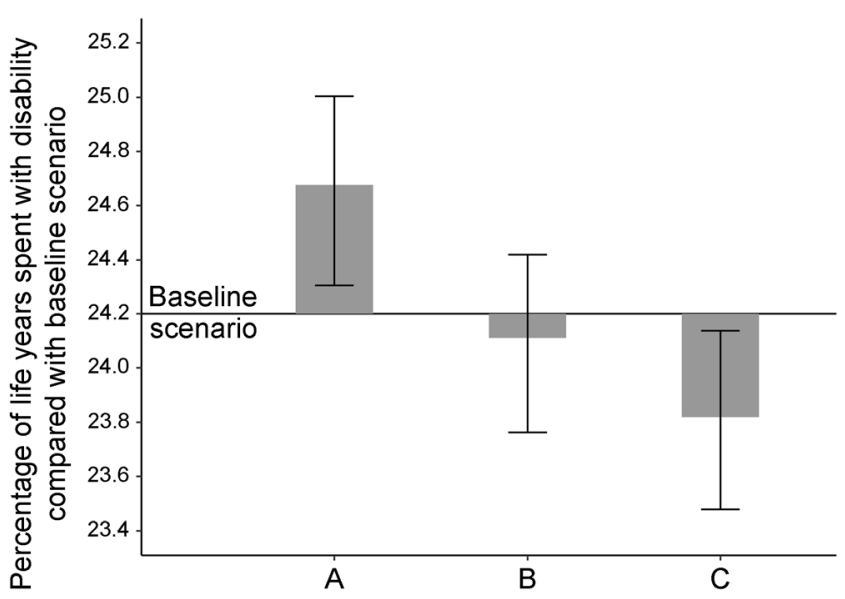

Fig. 3 Projected percentage of life years spent with disability in 2060 for England and Wales, population aged $\geq 65$. In the baseline scenario, $24.2 \%$ of life years were spent with disability (horizontal solid line). Bars represent corresponding proportions for scenarios A, B and C. Error bars represent $95 \%$ UIs. Scenarios: A, 49\% increase in diabetes by 2060; B, $20 \%$ increase in diabetes by $2060 ; C, 7 \%$ increase in diabetes by 2060

diseases. However, there is increasing evidence of the association between diabetes and dementia [4, 28-31].

Finally, all the disability caused by conditions other than dementia and CVD is aggregated into a single model state. As a consequence, the transition probabilities of moving from this state to other states are not specific for these conditions, but rather represent an average probability for all other causes of disability. Nevertheless, because the ELSA sample is representative for the population of England and Wales, the estimated combined probabilities should be generalisable.

\section{Public health implications}

Our findings have implications for the medium and long term. With a 35-year perspective we might expect a shift in the burden of diabetes consequences, from CVD towards a much broader range of sequelae including dementia. Our study suggests that preventing diabetes is important, not only for future CVD, but also for the dementia and disability burden. Moreover, as CVD mortality continues to decline, we expect a further shift in the burden of diabetes to non-cardiovascular complications. Contrary to some other diabetes complications, such as nephropathy or retinopathy, people with cognitive decline and dementia cannot be offered treatment to significantly slow down the progress of the disease. Since an older population structure is the leading reason for the increase in the dementia burden, it is reasonable to expect that dementia will increase as a share of diabetes complications.

Constraining the diabetes epidemic could reduce dementia incidence in future decades, however the effect is likely to be gradual. The risk of CVD improves almost immediately in response to interventions that decrease serum cholesterol, BP or smoking [32]. In contrast, our study suggests a much longer time lag between decreases in population rates of obesity and diabetes, and subsequent reductions in the dementia and disability burden.

Evidence suggests that upstream, structural interventions are far more powerful prevention strategies, and also more likely to reduce inequalities, than individual level agentic interventions [33]. Several UK policies now aim to halt the rise in obesity and diabetes prevalence, including the Calorie Reduction Programme, the Sugar Reduction Programme, marketing campaigns, the Soft Drinks Industry Levy and National Diabetes Prevention Programme [11, 12, 34]. In terms of individual level interventions, the English NHS Health Check programme for adults aged 40-74, which already includes $\mathrm{HbA}_{1 \mathrm{c}}$ or fasting blood glucose to identify diabetes in people with high BMI and/or BP, has recently been updated to include dementia advice. This may be useful for patient education as recent research suggested nearly half of adults over 50 did not know that a healthy lifestyle could help prevent dementia [35].

\section{Conclusions}

Future reductions in diabetes prevalence could reduce the burden of disability and dementia, however these reductions in dementia and disability burden will be observed in the mid to long term. Other preventative interventions targeting the shared determinants of these and other non-communicable diseases must be harnessed to stem the rising tide of dementia and disability in England.

Data availability The mortality rates data used in this analysis are available at the Office for National Statistics website: www.ons.gov.uk/ peoplepopulationandcommunity/populationandmigration/ populationprojections/methodologies/2016basednationalpopula tionprojectionsconsultationpapers.

Data on prevalence of obesity and diabetes used in this analysis are available at the NHS Digital website: https://digital.nhs.uk/data-andinformation/publications/statistical/health-survey-for-england/healthsurvey-for-england-2011-trend-tables.

ELSA data used in this analysis are available at UK Data Service: https://doi.org/10.5255/UKDA-SN-5050-16.

Funding This study was funded by the British Heart Foundation (grant numbers RG/13/2/30098, RG/16/11/32334). The funder had no role in study design, data collection and analysis, decision to publish or preparation of the manuscript.

Duality of interest HW reports grants from GlaxoSmithKline, outside the submitted work. All other authors declare there is no duality of interest associated with their contribution to this manuscript.

Contribution statement All authors made a substantial contribution to study conception and design, drafting the article and revising it critically for important intellectual content. EJB and $\mathrm{MO}^{\prime} \mathrm{F}$ developed the original idea. MG-C and PB designed and implemented the model, with input from MO'F, SA-A, MJS, JP-S, BC, HW and EJB. SA-A and HW prepared meta-analyses quantifying risk of developing dementia and disability in individuals with diabetes. SA-A, MJS, MG-C and PB analysed and prepared the data. All authors approved the final version of the manuscript. PB is the guarantor of this work. 


\section{References}

1. Ahmadi-Abhari S, Guzman-Castillo M, Bandosz P et al (2017) Temporal trend in dementia incidence since 2002 and projections for prevalence in England and Wales to 2040: modelling study. BMJ 358:j2856. https://doi.org/10.1136/bmj.j2856

2. GBD 2015 Disease and Injury Incidence and Prevalence Collaborators (2016) Global, regional, and national incidence, prevalence, and years lived with disability for 310 diseases and injuries, 1990-2015: a systematic analysis for the global burden of disease study 2015. Lancet 388(10053):1545-1602. https://doi. org/10.1016/S0140-6736(16)31678-6

3. Livingston G, Sommerlad A, Orgeta V et al (2017) Dementia prevention, intervention, and care. Lancet 390(10113):26732734. https://doi.org/10.1016/S0140-6736(17)31363-6

4. Gottesman RF, Albert MS, Alonso A et al (2017) Associations between midlife vascular risk factors and 25-year incident dementia in the Atherosclerosis Risk in Communities (ARIC) cohort. JAMA Neurol 74(10):1246-1254. https://doi.org/10.1001/jamaneurol. 2017.1658

5. Tuligenga RH, Dugravot A, Tabák AG et al (2014) Midlife type 2 diabetes and poor glycaemic control as risk factors for cognitive decline in early old age: a post-hoc analysis of the Whitehall II cohort study. Lancet Diabetes Endocrinol 2(3):228-235. https:// doi.org/10.1016/S2213-8587(13)70192-X

6. NHS Digital (2018) Statistics on Obesity, Physical Activity and Diet - England, 2018. Available from https://digital.nhs.uk/dataand-information/publications/statistical/statistics-on-obesityphysical-activity-and-diet/statistics-on-obesity-physical-activityand-diet-england-2018. Accessed 3 Jul 2018

7. GOV.UK (2015) Diabetes prevalence estimates for local populations. Available from: https://www.gov.uk/government/ publications/diabetes-prevalence-estimates-for-local-populations. Accessed 02 Nov 2017

8. NHS Digital (2015) Health Survey for England, 2015: Trend tables. Available from: https://digital.nhs.uk/data-and-information/ publications/statistical/health-survey-for-england/health-surveyfor-england-2011-trend-tables. Accessed 20 Nov 2017

9. Pedditzi E, Peters R, Beckett N (2016) The risk of overweight/ obesity in mid-life and late life for the development of dementia: a systematic review and meta-analysis of longitudinal studies. Age Ageing 45(1):14-21. https://doi.org/10.1093/ageing/afv151

10. Biessels GJ, Staekenborg S, Brunner E, Brayne C, Scheltens P (2006) Risk of dementia in diabetes mellitus: a systematic review. Lancet Neurol 5(1):64-74. https://doi.org/10.1016/S14744422(05)70284-2

11. Sood HS, Maruthappu M, Valabhji J (2015) The National Diabetes Prevention Programme: a pathway for prevention and wellbeing. $\mathrm{Br}$ J Gen Pract 65(636):336-337. https://doi.org/10.3399/ bjgp15X685537

12. GOV.UK. Health matters: preventing type 2 diabetes. In: www.gov. uk/government/publications/health-matters-preventing-type-2diabetes/health-matters-preventing-type-2-diabetes. Accessed 3 Jul 2018

13. Guzman-Castillo M, Ahmadi-Abhari S, Bandosz P et al (2017) Forecasted trends in disability and life expectancy in England and Wales up to 2025: a modelling study. Lancet Public Health 2(7): e307-e313. https://doi.org/10.1016/S2468-2667(17)30091-9

14. Eilers PHC, Marx BD (1996) Flexible smoothing with B-splines and penalties. Stat Sci 11(2):89-102

15. Currie ID, Durban M, Eilers PHC (2006) Generalized linear array models with applications to multidimensional smoothing. J R Stat
Soc Ser B Stat Methodol 68(2):259-280. https://doi.org/10.1111/j. 1467-9868.2006.00543.x

16. Currie ID, Durban M, Eilers PHC (2004) Smoothing and forecasting mortality rates. Stat Model Int J 4(4):279-298. https://doi.org/ 10.1191/1471082X04st080oa

17. Jorm A, Korten A (1988) Assessment of cognitive decline in the elderly by informant interview. Br J Psychiatry 152(2):209-213. https://doi.org/10.1192/bjp.152.2.209

18. Briggs A, Sculpher M (1998) An introduction to Markov modelling for economic evaluation. PharmacoEconomics 13(4):397-409. https://doi.org/10.2165/00019053-199813040-00003

19. Ford ES, Ajani UA, Croft JB et al (2007) Explaining the decrease in U.S. deaths from coronary disease, 1980-2000. N Engl J Med 356(23):2388-2398. https://doi.org/10.1056/ NEJMsa053935

20. Pearson-Stuttard J, Bandosz P, Rehm CD et al (2017) Reducing US cardiovascular disease burden and disparities through national and targeted dietary policies: a modelling study. PLoS Med 14(6): e1002311. https://doi.org/10.1371/journal.pmed.1002311

21. Beeri MS, Ravona-Springer R, Moshier E et al (2014) The Israel Diabetes and Cognitive Decline (IDCD) study: design and baseline characteristics. Alzheimers Dement 10(6):769-778. https://doi.org/ 10.1016/j.jalz.2014.06.002

22. WHO (2004) Comparative quantification of health risks. In: WHO Health statistics and information systems. Available from www. who.int/healthinfo/global_burden_disease/cra/en/. Accessed 16 Oct 2018

23. Zoungas S, Woodward M, Li Q et al (2014) Impact of age, age at diagnosis and duration of diabetes on the risk of macrovascular and microvascular complications and death in type 2 diabetes. Diabetologia 57(12):2465-2474. https://doi.org/10.1007/s00125014-3369-7

24. Barnes DE, Yaffe K (2011) The projected effect of risk factor reduction on Alzheimer's disease prevalence. Lancet Neurol 10(9):819 828. https://doi.org/10.1016/S1474-4422(11)70072-2

25. Okereke OI, Kang JH, Cook NR et al (2008) Type 2 diabetes mellitus and cognitive decline in two large cohorts of communitydwelling older adults. J Am Geriatr Soc 56(6):1028-1036. https:// doi.org/10.1111/j.1532-5415.2008.01686.x

26. Murray CJ, Lopez AD (1997) Alternative projections of mortality and disability by cause 1990-2020: Global Burden of Disease Study. Lancet 349(9064):1498-1504. https://doi.org/10.1016/ S0140-6736(96)07492-2

27. WHO (2016) Global report on diabetes. Available from www.who. int/diabetes/global-report/en/. Accessed 10 Jul 2018

28. Cukierman T, Gerstein HC, Williamson JD (2005) Cognitive decline and dementia in diabetes-systematic overview of prospective observational studies. Diabetologia 48(12):2460-2469. https:// doi.org/10.1007/s00125-005-0023-4

29. Gudala K, Bansal D, Schifano F, Bhansali A (2013) Diabetes mellitus and risk of dementia: a meta-analysis of prospective observational studies. J Diabetes Investig 4(6):640-650. https://doi.org/ 10.1111/jdi.12087

30. Ojo O, Brooke J (2015) Evaluating the association between diabetes, cognitive decline and dementia. Int J Environ Res Public Health 12(7):8281-8294. https://doi.org/10.3390/ijerph120708281

31. Ott A, Stolk RP, van Harskamp F, Pols HA, Hofman A, Breteler MM (1999) Diabetes mellitus and the risk of dementia: the Rotterdam Study. Neurology 53(9):1937-1942

32. Capewell S, O'Flaherty M (2011) Rapid mortality falls after riskfactor changes in populations. Lancet 378(9793):752-753. https:// doi.org/10.1016/S0140-6736(10)62302-1 
33. McGill R, Anwar E, Orton L et al (2015) Are interventions to promote healthy eating equally effective for all? Systematic review of socioeconomic inequalities in impact. BMC Public Health 15: 457. https://doi.org/10.1186/s12889-015-1781-7

34. GOV.UK (2018) Soft drinks industry levy comes into effect. Available from www.gov.uk/government/news/soft-drinksindustry-levy-comes-into-effect. Accessed 15 Jun 2018
35. GOV.UK (2018) Health, ageing and support survey: 2017. Available from www.gov.uk/government/publications/healthageing-and-support-survey-2017. Accessed 10 Jul 2018

Publisher's note Springer Nature remains neutral with regard to jurisdictional claims in published maps and institutional affiliations. 